\title{
THE WEYL ESSENTIAL SPECTRUM
}

\author{
RICHARD BOULDIN
}

Abstract. Using a modest geometric hypothesis the main theorem of these results classifies the Weyl essential spectrum and the Browder essential spectrum according to the standard terminology for the spectrum of a Hilbert space operator.

1. Introduction. The recent papers [1], [4], [6], [8] have classified the Weyl essential spectrum for various classes of operators on a Hilbert space. In [3] we classified the Wolf spectrum, the Weyl spectrum, and the Browder spectrum for a Hilbert space operator reduced by its finite dimensional geometric eigenspaces. We showed that our theorems contained the results in the above-mentioned papers and finally we listed a number of applications. Our purpose in the present note is to examine a condition which is alternative to the hypothesis that each finite dimensional geometric eigenspace reduces the operator. This alternative condition is properly weaker, although somewhat more complicated. We deduce a simplified form of the main result of [3] using this alternative hypothesis; neither do we repeat the applications of the main result nor do we relate the main theorem to the above-mentioned papers since that is the same as in [3]. We study the alternative hypothesis in order to show that it is properly weaker than the previous hypothesis and to show that it is easily verified.

2. Preliminaries. Throughout this note we shall use "operator" to mean a closed linear operator defined on a vector space which is dense in the fixed underlying Hilbert space $H$. If $T$ is such an operator and $H_{0}$ is a subspace of $H$ invariant under $T$ then $T / H_{0}$ denotes the restriction of $T$ to $H_{0}$. The conjugate of the complex number $z$ is written $z^{*}$ and we write the scalar operator $z I$ as simply $z$. We say $\lambda$ is an isolated point of $\sigma(T)$ to mean there is no sequence $\left\{\lambda_{n}: \lambda_{n} \neq \lambda, \lambda_{n} \in \sigma(T)\right\}$ which converges to $\lambda$ and we denote closure of the set $S$ by $S^{-}$.

An operator $T$ is said to be Fredholm if dimension [kernel $T$ ] $<\infty$, $T H$ is closed, and codimension $T H<\infty$. If $T$ is Fredholm then the

Presented to the Society, November 21, 1970; received by the editors April 28, 1970 and, in revised form, September 1, 1970.

AMS 1969 subject classifications. Primary 4615, 4710; Secondary 4748, 4730.

Key words and phrases. Essential spectrum, operator on a Hilbert space, eigenvalue, algebraic multiplicity, geometric multiplicity, Fredholm operator, index, closed range. 
index of $T$ is $[\operatorname{dim} \operatorname{ker} T$-codim $T H]$. We recall the following definitions of essential spectrum which have been given for closed operators in a Banach space. A point $\lambda$ from the spectrum of $T$, i.e. $\lambda \in \sigma(T)$, is in the essential spectrum of Wolf provided that either $(T-\lambda) H$ is not closed or else it has infinite codimension. The essential spectrum of Weyl is $\{\lambda \in \sigma(T):(T-\lambda)$ is not a Fredholm operator with index equal to 0$\}$; the essential spectrum of Browder is $\{\lambda \in \sigma(T)$ : either $(T-\lambda)$ is not a Fredholm operator with index equal to 0 or $\lambda$ is not an isolated point of $\sigma(T)\}$. If these three sets are ordered by inclusion then the above enumeration is clearly nondecreasing.

In order to save space we are going to give a name to the condition which is our basic hypothesis. The condition is the following: corresponding to $\lambda \in \sigma(T)$ there is a number less than 1 , say $\delta$, such that $|\langle f, g\rangle| \leqq \delta$ whenever $f \in \operatorname{ker}(T-\lambda),\|f\|=1=\|g\|$, and $g$ is an eigenvector for some eigenvalue distinct from $\lambda$. When the condition is satisfied we shall say "ker $(T-\lambda)$ is not an asymptotic eigenspace." Of course if the condition fails to hold then " $\operatorname{ker}(T-\lambda)$ is an asymptotic eigenspace."

3. Classification of essential spectrum. We prove a basic fact about the spectrum of a Hilbert space operator.

TheOREM 1. Let $T$ be an operator on $H$ such that $\operatorname{ker}(T-\lambda)$ is not an asymptotic eigenspace of $T$ and let $\left\{\lambda_{n}\right\}$ be a sequence such that $\lambda_{n} \neq \lambda, \lambda_{n} \in \sigma(T)$, and $\lambda_{n} \rightarrow \lambda$. If for each $n=1,2, \cdots$ there exists a unit vector $f_{n}$ such that $f_{n} \in \operatorname{ker}\left(T-\lambda_{n}\right)$ then $(T-\lambda) H$ is not closed.

Proof. Take a sequence of unit vectors such that $\left\|\left(T-\lambda_{n}\right) f_{n}\right\|$ $=0$ and decompose $f_{n}$ into $f_{n}^{\prime}+f_{n}^{\prime \prime}$ where $f_{n}^{\prime} \in \operatorname{ker}(T-\lambda)$ and $f_{n}^{\prime \prime} \in[\operatorname{ker}(T-\lambda)]^{\perp}$. If $P$ is the orthogonal projection onto $\operatorname{ker}(T-\lambda)$ then $f_{n}^{\prime}=P f_{n}$ and $f_{n}^{\prime \prime}=(I-P) f_{n}$. Because ker $(T-\lambda)$ is not an asymptotic eigenspace of $T$ for each $n$ and any unit vector $g \in P H$ we have $\left|\left\langle f_{n}, g\right\rangle\right| \leqq \delta$; in particular this is true for $g=f_{n}^{\prime}$, that is

$$
\begin{aligned}
\delta & \geqq\left|\left\langle f_{n}, f_{n}^{\prime}\right\rangle\right|=\left|\left\langle f_{n}, P f_{n}\right\rangle\right|=\left|\left\langle f_{n}, P^{2} f_{n}\right\rangle\right| \\
& =\left|\left\langle P f_{n}, P f_{n}\right\rangle\right|=\left\|f_{n}^{\prime}\right\|^{2} .
\end{aligned}
$$

Thus $\left\|f_{n}^{\prime \prime}\right\|^{2}=1-\left\|f_{n}^{\prime}\right\|^{2} \geqq 1-\delta>0$. Now we note the following simplifications

$$
\begin{aligned}
(T-\lambda) f_{n}^{\prime \prime} & =(T-\lambda)\left(f_{n}-f_{n}^{\prime}\right)=(T-\lambda) f_{n}, \\
\left\|(T-\lambda) f_{n}^{\prime \prime}\right\| & \leqq\left\|\left(T-\lambda_{n}\right) f_{n}\right\|+\left\|\left(\lambda_{n}-\lambda\right) f_{n}\right\| \\
& =\left\|\left(T-\lambda_{n}\right) f_{n}\right\|+\left|\lambda_{n}-\lambda\right| .
\end{aligned}
$$


Consequently $\left\|(T-\lambda) f_{n}^{\prime \prime}\right\| \rightarrow 0$ as $n \rightarrow \infty$. We may replace the original sequence $\left\{f_{n}\right\}$ with the sequence $\left\{f_{n}^{\prime \prime} /\left\|f_{n}^{\prime \prime}\right\|\right\}$ and thus we may assume that $\left\|(T-\lambda) f_{n}\right\| \rightarrow 0$ and $f_{n} \in[\operatorname{ker}(T-\lambda)]^{\perp}$.

It follows that $(T-\lambda)^{+}(T-\lambda) f_{n}=f_{n}$ where $(T-\lambda)^{+}$is the linear transformation inverse to $(T-\lambda)$ : $[\operatorname{ker}(T-\lambda)]^{\perp} \rightarrow[(T-\lambda) H]$-. If $(T-\lambda) H$ is closed then $(T-\lambda)^{+}$is bounded by application of the closed graph theorem, noting that $(T-\lambda) /[\operatorname{ker}(T-\lambda)]^{\perp}$ is a closed operator. Consequently if $(T-\lambda) H$ is closed then $(T-\lambda)^{+}$is bounded and $\left\|(T-\lambda)^{+}(T-\lambda) f_{n}\right\| \rightarrow 0$ since $\left\|(T-\lambda) f_{n}\right\| \rightarrow 0$. However this is nonsense since $\left\|(T-\lambda)+(T-\lambda) f_{n}\right\|=\left\|f_{n}\right\|=1$ and hence $(T-\lambda) H$ is not closed.

The following lemma is a special case of a well-known theorem.

Lemma 1. Let $\left\{\lambda_{n}\right\}$ be a sequence such that $\lambda_{n} \in \sigma(T), \lambda_{n} \neq \lambda$, and $\lambda_{n} \rightarrow \lambda$. If for each $n$ the operator $\left(T-\lambda_{n}\right)$ is not a Fredholm operator then $(T-\lambda)$ is not a Fredholm operator.

Proof. Because $\left(T-\lambda_{n}\right)$ converges to $(T-\lambda)$ in the operator norm we would contradict Theorem 5.17, p. 235 , of [7] if $(T-\lambda)$ were a Fredholm operator.

We now conclude our consideration of nonisolated points of the spectrum.

THEOREM 2. Let $T$ be an operator on $H$ such that $\operatorname{ker}(T-\lambda)$ is not an asymptotic eigenspace. If there exists a sequence, say $\left\{\lambda_{n}\right\}$, such that $\lambda_{n} \in \sigma(T), \lambda_{n} \neq \lambda$, and $\lambda_{n} \rightarrow \lambda$ then $(T-\lambda)$ is not a Fredholm operator.

Proof. For $\lambda_{n} \in \sigma(T)$ there are only three possibilities: (a) $\left(T-\lambda_{n}\right)$ is not one-to-one, (b) $\left(T-\lambda_{n}\right)$ is one-to-one but $\left(T-\lambda_{n}\right) H$ is not closed, (c) $\left(T-\lambda_{n}\right)$ is one-to-one with closed range but $\left[\left(T-\lambda_{n}\right) H\right]^{\perp}$ $\neq\{0\}$. Otherwise we could apply the closed graph theorem to see that $\left(T-\lambda_{n}\right)^{-1}$ is everywhere defined and bounded.

Now let $\left\{\lambda_{n}\right\}$ be any sequence such that $\lambda_{n} \in \sigma(T), \lambda_{n} \neq \lambda$, and $\lambda_{n} \rightarrow \lambda$. Either there is an infinite subset of $\left\{\lambda_{n}\right\}$ satisfying (a), in which case we apply Theorem 1 to see that $(T-\lambda) H$ is not closed, or else there is an infinite subset of $\left\{\lambda_{n}\right\}$ such that for each $\mu$ in that set $(T-\mu)$ satisfies either (b) or (c). If either (b) or (c) is satisfied by $(T-\mu)$ then this operator is not Fredholm and we may apply Lemma 1 to conclude that $(T-\lambda)$ is not Fredholm.

We are able to complete our consideration of isolated points of the spectrum with the following lemma. For a definition of algebraic multiplicity see pp. $178-181$ of [7].

LEMMA 2. Let $T$ be an operator on $H$ with $\lambda$ an isolated point of $\sigma(T)$. 
$(T-\lambda) H$ is closed with finite codimension if and only if $\lambda$ is an isolated eigenvalue with finite algebraic multiplicity.

Proof. See Lemma 3 and the second and third paragraphs of the proof of Theorem 3 in [3].

Now we can state the main result of this note.

THEOREM 3. If each finite dimensional subspace ker $(T-z)$, for any complex number $z$, is not an asymptotic eigenspace then the Weyl spectrum of $T$ coincides with the Browder spectrum of $T$ which coincides with the points of $\sigma(T)$ that are not isolated eigenvalues with finite algebraic multiplicity.

Proof. Apply Lemma 2 to any isolated point of $\sigma(T)$ and apply Theorem 2 to any nonisolated $\lambda$ such that $\operatorname{ker}(T-\lambda)$ is finite dimensional. If $\operatorname{ker}(T-\lambda)$ is infinite dimensional then obviously $(T-\lambda)$ is not Fredholm.

4. An asymptotic eigenspace. In this section we shall study the condition "ker $(T-\lambda)$ is not an asymptotic eigenspace." We shall show that our condition follows from several mild hypotheses. We recall that the angle between two subspaces $H_{1}$ and $H_{2}$ is the number $\theta \in[0, \pi]$ such that $\cos \theta=\sup \langle f, g\rangle$ where the supremum is taken over all unit vectors $f$ and $g$ such that $f \in H_{1}$ and $g \in H_{2}$. We say that two subspaces, $H_{1}$ and $H_{2}$, are complementary provided that $H$ $=H_{1} \oplus H_{2}$.

THEOREM 4. If $\operatorname{ker}(T-\lambda)$ has an invariant complementary subspace say $H_{1}$ with a positive angle between $H_{1}$ and $\operatorname{ker}(T-\lambda)$ then $\operatorname{ker}(T-\lambda)$ is not an asymptotic eigenspace.

Proof. Note that $\sigma(T)=\sigma(T / \operatorname{ker}(T-z)) \cup \sigma\left(T / H_{1}\right)$ and $\sigma(T / \operatorname{ker}(T-z))=\{z\}$. Let $\mu$ be an eigenvalue of $T$ distinct from $z$ and choose $g \in \operatorname{ker}(T-\mu)$. Write $g$ as $g^{\prime}+g^{\prime \prime}$ where $g^{\prime} \in \operatorname{ker}(T-z)$ and $g^{\prime \prime} \in H_{1}$ and note that $(T-\mu) g^{\prime} \in \operatorname{ker}(T-z)$ and $(T-\mu) g^{\prime \prime} \in H_{1}$. Since $(T-\mu) g=0$ it must be that $(T-\mu) g^{\prime}=-(T-\mu) g^{\prime \prime}$ is in the intersection of $\operatorname{ker}(T-z)$ and $H_{1}$; hence $(T-\mu) g^{\prime}=0=(T-\mu) g^{\prime \prime}$. If $g^{\prime} \neq 0$ then $\mu \in \sigma(T / \operatorname{ker}(T-z))$ which is a contradiction. Thus $g$ $=g^{\prime \prime} \in H_{1}$ and we conclude that $\operatorname{ker}(T-\mu) \subset H_{1}$. Since $H_{1}$ has a positive angle to $\operatorname{ker}(T-z)$ we conclude that $\operatorname{ker}(T-\lambda)$ is not an asymptotic eigenspace.

COROLlaRy 1. If $\operatorname{ker}(T-\lambda)$ reduces $T$ then it is not an asymptotic eigenspace. 
The next theorem will justify the terminology "asymptotic eigenspace."

THEOREM 5. If $\operatorname{ker}(T-\lambda)$ is a finite dimensional asymptotic eigenspace then there exists a sequence of eigenvalues $\left\{\lambda_{n}\right\}$ with $\lambda_{n} \neq \lambda$ and a sequence of unit vectors $\left\{g_{n}\right\}$ with $g_{n} \in \operatorname{ker}\left(T-\lambda_{n}\right)$ such that $\left\{g_{n}\right\}$ converges in norm to a unit vector $f$ where $f \in \operatorname{ker}(T-\lambda)$. Furthermore, if $T$ is bounded then $\lambda_{n} \rightarrow \lambda$.

Proof. Because $(T-\lambda)$ is an asymptotic eigenspace for each $n$ we can find an eigenvalue $\lambda_{n}$ and unit vectors $g_{n} \in \operatorname{ker}\left(T-\lambda_{n}\right)$, $f_{n} \in \operatorname{ker}(T-\lambda)$ such that $\left\langle g_{n}, f_{n}\right\rangle \geqq 1-1 / n$. Since $\left\langle g_{n}, f_{n}\right\rangle \leqq\left\|g_{n}\right\|\left\|f_{n}\right\|$ we see that $\left\langle g_{n}, f_{n}\right\rangle \rightarrow 1$; using the fact that $\left\|g_{n}\right\|^{2}=1=\left\|f_{n}\right\|^{2}$ we conclude that $\left\langle g_{n}, g_{n}\right\rangle-\left\langle g_{n}, f_{n}\right\rangle=\left\langle g_{n}, g_{n}-f_{n}\right\rangle \rightarrow 0$ and $\left\langle f_{n}, g_{n}\right\rangle-\left\langle f_{n}, f_{n}\right\rangle$ $=\left\langle f_{n},\left(g_{n}-f_{n}\right)\right\rangle \rightarrow 0$. Thus $\left\|g_{n}-f_{n}\right\|^{2}$ converges to 0 . Because the unit ball in ker $(T-\lambda)$ is compact $\left\{f_{n}\right\}$ has a subsequence converging to some $f \in \operatorname{ker}(T-\lambda)$. Clearly $\left\{g_{n}\right\}$ converges to $f$ and if $T$ is bounded then $T g_{n}$ converges to $T f$ and $\lambda_{n}=\left\langle T g_{n}, g_{n}\right\rangle$ converges to $\lambda=\langle T f, f\rangle$.

This gives rise to another condition which is sufficient for $\operatorname{ker}(T-\lambda)$ not to be a finite dimensional asymptotic eigenspace.

Corollary 2. Let $T$ be a bounded operator. If $\lambda$ is not a limit point for the set of eigenvalues of $T$ then $\operatorname{ker}(T-\lambda)$ is not an asymptotic eigenspace.

The final two corollaries were called to the author's attention by the referee.

COROLlaRY 3. If $T$ has the property that $\operatorname{ker}(T-\lambda)$ is orthogonal to $\operatorname{ker}(T-\mu)$ whenever $\lambda \neq \mu$ then $T$ has no asymptotic eigenspaces.

COROLLARY 4. If $T$ has the property that the numerical radius of any restriction of $T$ to an invariant subspace is equal to the spectral radius of that restriction then $T$ has no asymptotic eigenspaces.

Proof. Use Lemma 5, p. 191, of [2] along with Corollary 3 above.

REMARK. We note that Theorem 1 is not necessarily true when $\operatorname{ker}(T-\lambda)$ is an asymptotic eigenspace. Let $S^{*}$ be the adjoint of the unilateral shift and recall that each $\lambda$ such that $|\lambda|<1$ is a simple eigenvalue for $S^{*}$ although $S^{*} H$ is clearly equal to $H$ and thus $S^{*} H$ is closed.

\section{BiBLIOGRAPHY}

1. S. K. Berberian, An extension of Weyl's theorem to a class of not necessarily normal operators, Michigan Math. J. 17 (1969), 273-279, MR 40 \#3335. 
2. S. K. Berberiaw, Some conditions on an operator implying normality, Math. Ann. 184 (1970), 188-192.

3. R. H. Bouldin, Essential spectrum for a Hilbert space operator (to appear).

4. L. A. Coburn, Weyl's theorem for nonnormal operators, Michigan Math. J. 13 (1966), 285-288. MR 34 \#1846.

5. P. R. Halmos, $A$ Hilbert space problem book, Van Nostrand, Princeton, N. J., 1967. MR 34 \#8178.

6. V. Istrătescu, Weyl's theorem for a class of operators, Rev. Roumaine Math. Pures Appl. 13 (1968), 1103-1105. MR 38 \#6381.

7. T. Kato, Perturbation theory for linear operators, Die Grundlehren der math. Wissenschaften, Band 132, Springer-Verlag, New York, 1966. MR 34 \#3324.

8. J. I. Nieto, On the essential spectrum of symmetrizable operators, Math. Ann. 178 (1968), 145-153. MR 38 \#1544.

University of Georgia, Athens, Georgia 30601 